\title{
Contra las culturas del acoso en la arqueología: Enfoques socioambientales y basados en el trauma para la transformación disciplinaria
}

\author{
Barbara L. Voss
}

\begin{abstract}
Este artículo es el segundo de una serie de dos partes que analiza la investigación actual sobre el acoso en la arqueología. Estudios cualitativos y cuantitativos, como también relatos de activistas y testimonios de sobrevivientes, han establecido que el acoso ocurre a tasas epidémicas en la arqueología. Estos estudios han identificado patrones clave en relación al acoso y señalan posibles intervenciones para prevenirlo, para apoyar a les sobrevivientes y para responsabilizar a les perpetradores. Este artículo discute cinco obstáculos clave a superar en la cultura disciplinaria de la arqueología: normalización, prácticas excluyentes, confraternización, gatekeeping e impedimentos para la presentación de denuncias. Con base en dos paradigmas de salud pública — el modelo socioambiental y los enfoques basados en el trauma - se identifican intervenciones potenciales aplicables a todos los niveles de la práctica arqueológica: individual, relacional, organizacional, comunitario y social.
\end{abstract}

Palabras clave: acoso y agresión sexual en la arqueología, discriminación, medidas de prevención, modelo socioambiental, atención enfocada en el trauma

This article is the second in a two-part series that analyzes current research on harassment in archaeology. Both qualitative and quantitative studies, along with activist narratives and survivor testimonials, have established that harassment is occurring in archaeology at epidemic rates. These studies have also identified key patterns in harassment in archaeology that point to potential interventions that may prevent harassment, support survivors, and hold perpetrators accountable. This article reviews five key obstacles to change in the disciplinary culture of archaeology: normalization, exclusionary practices, fraternization, gatekeeping, and obstacles to reporting. Two public health paradigms-the social-environmental model and traumainformed approaches - are used to identify interventions that can be taken at all levels of archaeological practice: individual, relational, organizational, community, and societal.

Keywords: harassment and sexual assault in archaeology, discrimination, prevention measures, social-environmental model, trauma-informed care

Nota de les editores. Barbara Voss ha publicado dos artículos en American Antiquity (volumen 86). Dada la importancia del tema y el interés de la SAA, de Cambridge University Press, de les editores de American Antiquity, Latin American Antiquity y de la propia autora en que estos artículos estén disponibles lo más ampliamente posible, nos complace publicar en Latin American Antiquity las traducciones oficiales al español de ambos artículos, que además incorporan el uso de lenguaje inclusivo. Al traducir este artículo, se utilizó lenguaje inclusivo para referirse a las personas cuyo género es no binario o no se conoce, y en el caso de grupes se utilizó lenguaje neutro cuando había más de un género. De esta manera, la Revista avala la utilización de diferentes formas de lenguaje inclusivo, no sexista y no discriminatorio. Las citas textuales que estaban originalmente en inglés se han traducido para esta publicación. Las versiones en inglés y en español son de libre acceso.

[Advertencia de contenido: Este artículo discute el acoso y la discriminación en la arqueología, e incluye relatos de agresión sexual.]

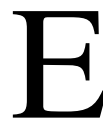
ste artículo es el segundo de una serie de dos partes que revisa y analiza la investigación que se ha hecho sobre el acoso en

Barbara L. Voss — (bvoss@ stanford.edu) Department of Anthropology, Stanford University, Stanford, CA, USA

Latin American Antiquity 33(1), 2022, pp. 1-19

Copyright (C) The Author(s), 2021. Published by Cambridge University Press on behalf of the Society for American Archaeology. This is an Open Access article, distributed under the terms of the Creative Commons AttributionNonCommercial-NoDerivatives licence (https://creativecommons.org/licenses/by-nc-nd/4.0/), which permits non-commercial re-use, distribution, and reproduction in any medium, provided the original work is unaltered and is properly cited. The written permission of Cambridge University Press must be obtained for commercial re-use or in order to create a derivative work. doi:10.1017/laq.2021.83 
la arqueología y otros campos relacionados. Teniendo en consideración que el primer artículo (Voss 2021) ha documentado el alcance del problema, éste describe soluciones basadas en evidencia que pueden acabar con las culturas del acoso en la arqueología. Los enfoques aquí descritos se basan en dos paradigmas de salud pública: el modelo socioambiental y la atención enfocada en el trauma. Este trabajo refleja mi experiencia de 35 años en la arqueología, en roles tanto de estudiante como de profesional en gestión de recursos culturales (CRM), en museos, y en el mundo académico. Aunque el acoso ocurre en la arqueología a tasas epidémicas, existen acciones claves que pueden prevenirlo o detenerlo tempranamente en caso de que ya haya occurrido y así reducir su impacto en les sobrevivientes. Es más probable que las intervenciones tengan éxito cuando se desarrollan en conjunto con sobrevivientes y otres arqueólogues vulnerables, cuando se aprovechan las capacidades organizativas, y cuando se basan en valores fundamentales comunes de colaboración y compañerismo en la arqueología.

\section{Lo que sabemos ahora sobre el acoso en la arqueología}

El término "acoso" se refiere a una amplia gama de prácticas discriminatorias e ilegales que suceden en espacios educativos y laborales. El acoso incluye comportamientos tanto físicos como no físicos que conllevan hostilidad, objetivación, exclusión o un estatus de segunda clase basado en el género percibido o en la orientación sexual de la persona atacada. El acoso no físico "puede provocar el mismo nivel de resultados profesionales y psicológicos negativos como ... . [los] casos de coacción sexual" (National Academies of Sciences, Engineering, and Medicine 2018). La identidad de género, la orientación sexual, la edad, la raza, la etnia, la nacionalidad, el origen de clase, lo queer o la discapacidad de una persona pueden aumentar su vulnerabilidad al acoso (Crenshaw 1991). Cada vez se utiliza más el término "acoso basado en la identidad" para abarcar una amplia gama de comportamientos discriminatorios y predatorios.

La lucha contra el acoso en la arqueología se remonta a finales del siglo diecinueve. Desde la década de 1970, la investigación sobre la igualdad de género ha identificado claramente al acoso como un factor importante que afecta de manera negativa a arqueólogues mujeres, LGBTQIA $+{ }^{1}$ y de color (Nelson et al. 1994). Durante las décadas de 2000 y 2010, muchas sociedades profesionales dentro de la arqueología publicaron declaraciones contundentes en contra del acoso. Lamentablemente, estas políticas por sí solas no han resultado efectivas para prevenir el aumento del acoso. Investigaciones recientes muestran que entre el 15 y el 46 por ciento de los hombres, y entre el 34 y el 75 por ciento de las mujeres han sufrido acoso en una o más ocasiones durante sus carreras. Entre el 5 y el 8 por ciento de los hombres y entre el $15 \mathrm{y}$ el 26 por ciento de las mujeres reportan contacto sexual no deseado, incluyendo agresión sexual (Voss 2021:Tabla 1).

Les investigadores identificaron los siguientes ocho patrones de acoso dentro de los lugares de trabajo arqueológicos y sus entornos de aprendizaje (Bradford y Crema 2020; Clancy et al. 2014; Coto-Sarmiento et al. 2018; Heath-Stout 2019; Hodgetts et al. 2020; Jalbert 2019; Meyers et al. 2015, 2018; Nelson et al. 2017; Radde 2018; Rocks-Macqueen 2018; VanDerwarker et al. 2018):

(1) El acoso ocurre en los sectores arqueológicos (CRM, agencias gubernamentales, museos, patrimonio y academia) y en todos sus entornos (sitios de campo, laboratorios, aulas, oficinas, museos, conferencias).

(2) Les arqueólogues son acosades con más frecuencia por otres arqueólogues, a menudo por miembros de su propio equipo de investigación.

(3) El acoso se dirige generalmente a arqueólogues en puestos en niveles más bajos.

(4) En general, las arqueólogas mujeres son más acosadas por hombres y por supervisores.

(5) Los arqueólogos hombres son acosados tanto por hombres como por mujeres en proporciones prácticamente iguales, y en general son acosados más por sus compañeres que por sus supervisores.

(6) Las arqueólogas mujeres tienen más probabilidades que los arqueólogos hombres de ser acosadas debido a su estatus familiar.

(7) Arqueólogues de color, de minorías étnicas, no binarios, LGBTQIA+ y arqueólogues 
con discapacidades reportan acoso a tasas más altas que el promedio.

(8) El acoso en la arqueología se aprende intergeneracionalmente a través de experiencias profesionales tempranas y de arqueólogues con mayor experiencia que alientan o presionan a les que tienen menos experiencia para que participen en conductas de acoso.

Las investigaciones y los testimonios han documentado los impactos negativos del acoso. Les sobrevivientes reportan que se "sienten 'vulnerables', 'impotentes', 'sin control', 'aislades' o como "presas"” (Nelson et al. 2017:714). A menudo se vuelven insegures acerca de sus propias habilidades y su futuro profesional (Heath-Stout 2019; Meyers et al. 2015:28). Según Nelson y colaboradores (2017:715), "El esfuerzo de procesar y tomar decisiones que implica la negociación de un ambiente hostil de trabajo y el mantenimiento del empleo puede ser agotador y conducir a una reducción de la salud física y mental." Esta carga cognitiva y psicológica afecta el tiempo, la energía y la atención en las actividades profesionales, agravando el impacto inicial del acoso y, en algunos casos, expulsando a les arqueólogues del campo (Clancy et al. 2014; Heath-Stout 2019; Nelson et al. 2017).

\section{Barreras para el cambio}

¿Por qué ha persistido el acoso en la arqueología? Existen cinco barreras clave para el cambio: normalización, prácticas de exclusión, confraternización, gatekeeping (un modismo inglés para referirse a alguien que controla el acceso a oportunidades profesionales) y obstáculos para la presentación de denuncias.

\section{Normalización}

En la investigación realizada a través de una encuesta sobre acoso, les encuestades "identificaron regularmente al acoso como parte de la 'cultura' de la arqueología” (Radde 2018:248-249) y expresaron que el acoso es "uno de los comportamientos tácitos, pero socialmente esperado en los lugares de trabajo arqueológicos" (Meyers et al. 2018:277; énfasis en el original). En suma, sostuvieron "que [el acoso] es algo que ocurre y que es normal" (Coto-Sarmiento et al. 2018:33).
Como muches arqueólogues, yo fui socializada para aceptar el acoso como algo normal y repetí de manera inconsciente algunas de las actitudes y comportamientos que presencié al principio de mi carrera. Por ejemplo, algunes supervisores se burlaban regularmente de mí, así que cuando yo me convertí en supervisora, a veces me burlaba de mis subordinades y alentaba a otres a unirse, olvidando lo incómodo que era ser el blanco de las bromas. También llegué a tolerar el comportamiento inapropiado. Por ejemplo, en un proyecto, une subordinade usaba regularmente un lenguaje sexualizado cuando se dirigía a otres miembros del equipo. No me gustó y me di cuenta de que otres miembros del proyecto también se sentían incómodes. Pero a pesar de mi posición como supervisora, en ese momento me sentí incapaz de manejar directamente la conducta inapropiada de le subordinade.

La normalización del acoso también afectó mi estilo de asesoría. Hasta hace sólo unos años, cuando otres arqueólogues se me acercaron para pedirme consejo sobre cómo manejar situaciones de acoso, generalmente les aconsejaba ser fuertes y perseverar en lugar de desafiar o denunciar directamente a sus acosadores. Esta era la manera como yo había sobrevivido a lo largo de mi carrera y repetía los consejos que yo había recibido de mis mentores. Mirando hacia atrás, reconozco mis comportamientos pasados en lo que van der Vaart-Verschoof (2019) describe como

la profesora dura ... Por lo general, son mujeres de 50 o 60 años que llegaron a la academia y la arqueología en una época en que las cosas eran mucho peores para las mujeres. Tenían que luchar por todo $\mathrm{y}$, de manera completamente injusta, por cierto, tenían que demostrar su valor a un nivel mucho más alto que sus compañeros hombres. Y por alguna razón, he descubierto que muchas de ellas al parecer son aún más duras con mujeres jóvenes y las someten a un nivel de exigencia mucho más alto.

Me duele reflexionar sobre cómo fui partícipe en la reproducción y la normalización del acoso en la arqueología. Investigaciones recientes muestran que no estoy sola: las culturas del acoso en la arqueología se aprenden y se reproducen intergeneracionalmente (Heath-Stout 2019:283). 
Entender este proceso de normalización nos ayuda a darnos cuenta de la importancia que tiene la participación de cada arqueólogue en la lucha contra el acoso.

\section{Prácticas de exclusión}

La disciplina como un todo: blanca, de clase media, heterosexual, del primer mundo y sin discapacidad [Wylie y Nelson 1994:xi].

La arqueología expresa un régimen de género que valoriza todo lo relacionado con el varón que es activo fisicamente y activamente heterosexual, o quizás más específicamente, todo lo relacionado con un determinado tipo de masculinidad [Moser 2007:259].

El trabajo de campo es un entorno de bajo riesgo para les agresores . . y y un entorno de alto riesgo para las personas marginadas ... [con] mayor riesgo para individuos no hombres y no heterosexuales [Bradford y Crema 2020:2].

Los estereotipos de arqueólogos hombres, blancos, arriesgados y aventureros fomentan la creencia ampliamente compartida, aunque a menudo tácita, de que realizar una investigación arqueológica es más importante que la seguridad personal o comunitaria. Es más, se piensa que los riesgos incurridos agregan valor a la investigación. Esta actitud "incluye nociones románticas sobre aventura, resistencia física y alcoholismo . . . Entonces, todes les arqueólogues deben mostrar actitudes de masculinidad en los ambientes de campo, independientemente de su sexo biológico, para ser profesionales ideales y exitoses" (Geller 2016:160). En consecuencia, les arqueólogues que se resistieron al acoso o lo reportaron han sido "ridiculizades" y "estereotipades como personas histéricas, débiles, ingenuas y sin humor" (Wylie 1994). Cuando asumir riesgos es una norma disciplinaria, denunciar el acoso expone a les denunciantes a un mayor estigma y exclusión.

\section{Fraternización}

En los contextos educativos y profesionales la fraternización se refiere a las relaciones románticas y sexuales entre colegas. La fraternización tiene una larga historia en la arqueología. Inicialmente, el matrimonio con un arqueólogo hombre cisgénero era una de las pocas carreras disponibles para las arqueólogas mujeres. Desde la década de 1970, los nombramientos conjuntos de parejas de arqueólogues se han promovido activamente como una forma de mejorar la equidad de género. En el CRM del sector privado en los Estados Unidos, la preferencia del gobierno de contratar empresas que sean propiedad de mujeres ha incentivado la creación de compañías de propiedad conjunta por parte de parejas de arqueólogues heterosexuales. Todas estas medidas han intensificado la dependencia que tienen las carreras de las mujeres en la arqueología hacia las relaciones íntimas con arqueólogos hombres.

Incluso entre arqueólogos del mismo rango, confraternizar puede generar conflictos de intereses. El favoritismo real o percibido de une arqueólogue hacia su pareja puede resultar en acoso indirecto hacia los demás y generar un ambiente de trabajo o aprendizaje hostil. Les subordinades pueden sentirse doblemente reacies a denunciar el acoso si la pareja de la persona que comete el abuso tiene un papel en la investigación, o si existe la posibilidad de represalias por parte de la pareja no acosadora.

El problema de la confraternización se agudiza cuando se generan relaciones íntimas entre supervisores y subordinades. Los testimonios de les sobrevivientes y los relatos de espectadores reflejan que, sean cuales sean las intenciones de le arqueólogue sénior, les subordinades muchas veces interpretan las invitaciones y avances dentro de un contexto cargado de poder debido a su relación profesional:

Conseguí un empleo dirigiendo el laboratorio de arqueología, en donde aprendí a hacer varios análisis de la asistente de la directora del laboratorio. Después de un año de sentirme solo y de pobreza, cedí a sus insinuaciones. Cocinó para mí, me compró regalos, me dio un trabajo [She 2000:170].

El profesor ... me preguntó si le estaba practicando sexo oral a mi supervisor ... y luego se me insinuó . . . Como él era profesor y yo [era] "sólo" una estudiante, sentí que no 
había nada que pudiera hacer con respecto a su comportamiento tremendamente inapropiado [van der Vaart-Verschoof 2019].

Sentí que no podía negarme sin poner en peligro mi calificación o mi recomendación . . . He sufrido mucho por haber participado en relaciones sexuales no deseadas y me deprimí enormemente [Estudiante anónime, citado en Bikales 2020].

Todavía tenemos que desarrollar mejores prácticas para abordar el impacto de la fraternización en la cultura disciplinaria de la arqueología. Adoptar principios de no confidencialidad y recusación adecuada sería un buen punto de partida para reducir los prejuicios reales y percibidos relacionados con las relaciones íntimas en entornos de aprendizaje y en lugares de trabajo arqueológicos. Se podría mitigar la posibilidad de sesgo si se evita colocar en los puestos de supervisión y evaluación de un proyecto a las parejas de les arqueólogues involucrades y, cuando sea posible, se asignen a equipos o departamentos diferentes. Los desequilibrios de poder y el riesgo de incurrir en favoritismo o coerción en las relaciones íntimas entre arqueólogues sénior y junior "hacen que sea difícil determinar si estas relaciones son consensuales" (HeathStout 2019:280). Se requieren políticas claras contra la fraternización entre arqueólogues de diferentes rangos para transformar las culturas arqueológicas del acoso.

\section{Gatekeeping}

Les acosadores suelen ser gatekeepers que tienen la capacidad de restringir el acceso de les investigadores a la comunidad y a la información. En tales casos, les acosadores a menudo son conscientes de su poder y autoridad ante le investigadore y su trabajo, y asumen esa dependencia [Kloß 2017:405].

La investigación arqueológica requiere colaboración, así como permisos, financiamiento, laboratorios, colecciones y equipo especializado. Como arqueólogues, dependemos de les gatekeepers. Elles pueden aprobar o denegar peticiones y solicitudes que son necesarias para acceder a los recursos esenciales de nuestras carreras. Las disciplinas como la arqueología que son "jerárquicas, con fuerte dependencia de los niveles superiores ... son más propensas a fomentar y mantener el acoso sexual" (Johnson et al. 2018:4). Aunque la mayoría de les arqueólogues ejercen su función como gatekeepers con integridad, es necesario salvaguardar y garantizar que les subordinades puedan reportar el mal comportamiento de algunes sin arriesgar sus carreras.

\section{Obstáculos para denunciar}

Los procedimientos de denuncia actuales a menudo aumentan la vulnerabilidad de les sobrevivientes en lugar de protegerles. El bajo número de denuncias "sugiere que nuestra cultura disciplinaria no fomenta la denuncia, que denunciar el acoso no está socialmente sancionado o que el miedo a las represalias es un factor importante" (Radde 2018:244). Como observan Hodgetts y colaboradores (2020:37),

La mayoría de estos incidentes tienen lugar dentro de comunidades pequeñas (equipos de campo, departamentos universitarios, oficinas de CRM) que están estructuradas jerárquicamente. Debido a que quien perpetra las experiencias negativas es a menudo alguien con autoridad, reportar podría resultar en la pérdida del trabajo o en un acceso reducido a oportunidades profesionales.

En consecuencia, sólo un número reducido de arqueólogues que ha sufrido acoso reporta a sus acosadores. Les encuestades (Radde 2018:244, $249,250)$ hicieron las siguientes declaraciones:

- "Estamos demasiado preocupades por perder nuestras carreras como para hacer un reporte formal."

- "En ese momento no estaba en una posición para desafiar, y [yo] temía ser removide de los proyectos."

- "Sabía que, si denunciaba, me harían la vida imposible."

- "A veces es más problema de lo que se gana, y no quieres preocuparte por las represalias o por ser etiquetada como una persona 'problemática'."

Varias veces, denuncié el acoso a mis supervisores directos, quienes estaban obligades por ley y por política institucional a tomar medidas formales sobre las quejas. Ningune lo hizo. Une me 
regañó: "Si planeas huir cada vez que un arqueólogo sexista trata de evitar que alcances tus metas, es mejor que renuncies ahora mismo." Otre supervisore advirtió que la denuncia formal me expondría a represalias. En la mayoría de los casos, sin embargo, mis reportes simplemente fueron ignorados.

Dada la ausencia de canales efectivos para la denuncia formal, las víctimas de acoso recurren cada vez más a las redes sociales, a los testimonios anónimos y a la acción colectiva como medios para "distribuir el riesgo de denuncia" (Awesome Small Working Group [ASWG] 2019:18). El activismo digital y la denuncia colectiva están forzando respuestas institucionales y generando apoyo para sobrevivientes individuales.

\section{Enfoques de salud pública para alterar las culturas del acoso}

Los patrones generalizados de acoso en la arqueología y las barreras al cambio indican que un enfoque desde la salud pública puede ser útil. Esta perspectiva no exime de responsabilidad a las personas, pero sugiere que las iniciativas para reducir o eliminar el acoso serán más efectivas si se abordan los factores que lo causan en múltiples niveles. El resto de este artículo adapta dos enfoques basados en la evidencia el modelo socioambiental y el modelo enfocado en el trauma- para identificar intervenciones que pueden prevenir el acoso en la arqueología, apoyar a les sobrevivientes y responsabilizar a les acosadores.

El modelo socioambiental ve los problemas de salud pública como "la consecuencia del desarrollo de una causalidad recíproca en múltiples niveles de influencia individual y ambiental" (Richard et al. 2011:309; Figura 1). A nivel individual, la historia personal y los comportamientos actuales de cada arqueólogue contribuyen a perpetuar o interrumpir el acoso. El nivel relacional reconoce que los pensamientos, creencias y acciones de cada arqueólogue están influenciados por sus interacciones con otres arqueólogues. El nivel organizacional reconoce la importancia de las instituciones que brindan capacitación, empleo, recursos y comunicación profesional en el establecimiento de normas de conducta profesional. El nivel comunitario se refiere a las interacciones entre esas organizaciones. El nivel social incluye leyes, políticas y regulaciones gubernamentales, desigualdades económicas entre individuos, organizaciones y comunidades, creencias, actitudes y normas culturales ampliamente arraigadas, y cualquier otro factor de amplio alcance que "ayude a crear un clima en el que [el acoso] sea fomentado o inhibido" (Centers for Disease Control and Prevention 2019).

Al igual que el modelo socioambiental, los enfoques basados en el trauma son modelos de intervención basados en evidencia que apuntan a abordar la discriminación, el acoso y la violencia sexual en un marco de salud pública. Los enfoques basados en el trauma se desarrollaron por primera vez hace más de 40 años en centros de crisis para víctimas de violación, dentro de los movimientos sociales contra la violencia doméstica y en redes de sobrevivientes. Estos modelos de intervención han sido avalados por investigaciones de salud pública y ahora son el estándar ampliamente adoptado y respaldado por comunidades profesionales médicas y legales, así como por agencias gubernamentales de salud (National Sexual Assault Coalition Resource Sharing Project and National Sexual Violence Resource Center [NSACRSP/NSVRC] 2017:5; Substance Abuse and Mental Health Services Organization [SAMHSA] 2014:5).

El trauma es una respuesta que "resulta de un evento, serie de eventos o conjunto de circunstancias que se experimentan . . . como dañinas física y emocionalmente" (SAMHSA 2014:7). Comienza cuando un evento o experiencia abruma los mecanismos normales de supervivencia de un individuo o un grupo (NSACRSP/ NSVRC 2017:5). El trauma primario ocurre cuando el evento traumático es experimentado directamente por el individuo o el grupo; el trauma secundario ocurre entre individuos o grupos que presencian eventos traumáticos o que están apoyando a miembros traumatizados de su comunidad. Además, el trauma es multiescala: "Los traumas pueden afectar a personas, familias, grupos, comunidades, culturas específicas y generaciones" (CSAT-US 2014:7).

Un enfoque basado en el trauma depende de tres principios básicos. En primer lugar, es más probable que un individuo o un grupo que sufrió 


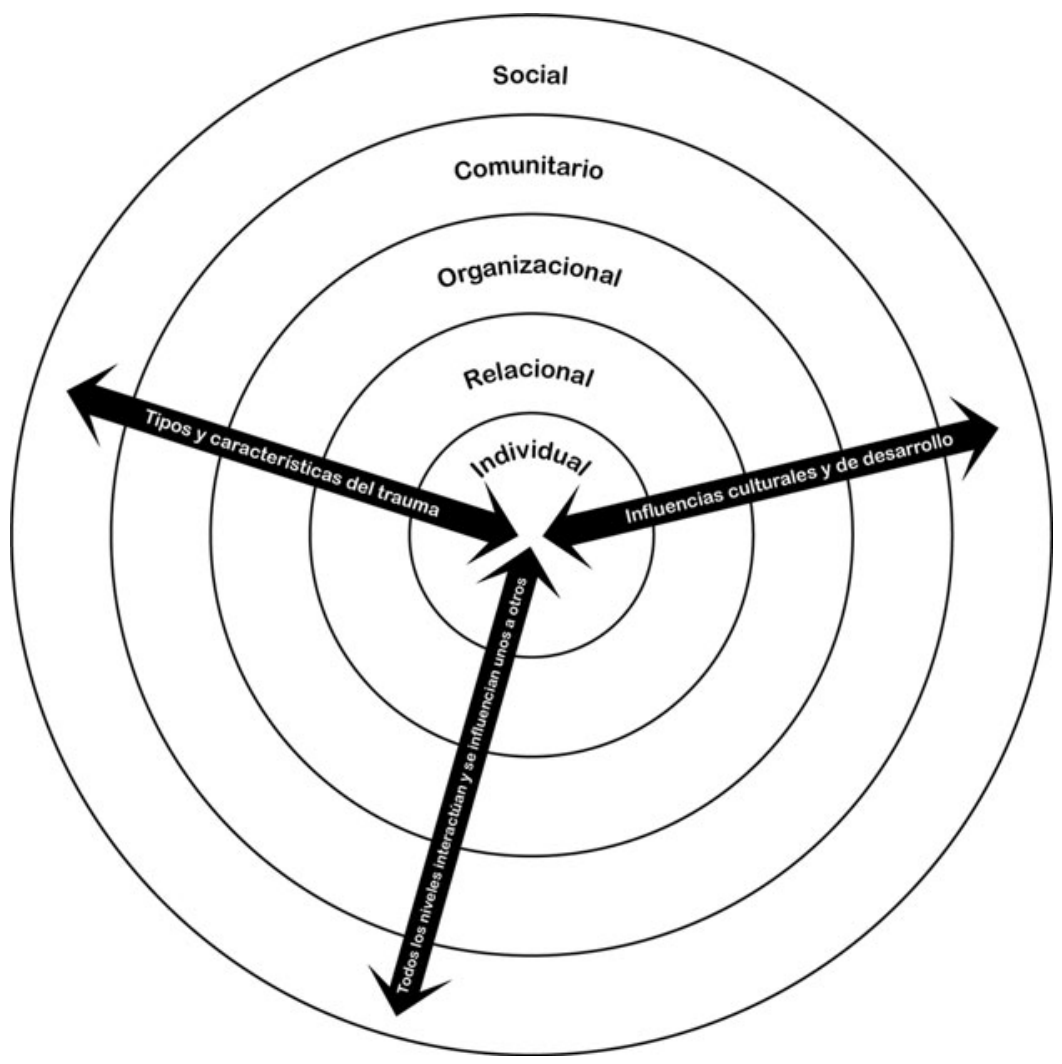

Figura 1. Modelo basado en el trauma informado y socio ambiental de salud pública. Adaptado del Center for Substance Abuse Treatment (CSAT-US; 2014:15). Gráfico realizado por Katie Johnson-Noggle.

o cometió acoso anteriormente tenga un historial de trauma. Ahora sabemos que el acoso y la agresión sexual ocurren a tasas epidémicas en la arqueología. Además, les arqueólogues, como miembros de una sociedad más amplia, están expuestes al acoso, la agresión sexual y otras formas de trauma en otros aspectos de su vida diaria. Debido a que la mayoría de les arqueólogues probablemente han experimentado o presenciado acoso, "no necesariamente necesitamos interrogar a las personas sobre sus experiencias; más bien, debemos asumir que pueden tener este historial y actuar en consecuencia" (Tello 2018).

En segundo lugar, sin una intervención deliberada, las actitudes sociales y los procedimientos organizativos que minimizan o ignoran la experiencia traumática pueden volver a traumatizar inadvertidamente a quienes se han visto afectados por el acoso. La re-traumatización es "cualquier situación o entorno que se asemeje al trauma de una persona, de forma literal o simbólica, que luego desencadene sentimientos y reacciones difíciles asociados con el trauma original" (University at Buffalo, Buffalo Center for Social Research 2021). Este tipo de re-traumatización se da en los casos cuando los relatos del trauma de les sobrevivientes son trivializados o descartados, cuando les sobrevivientes tienen poco o ningún control sobre los detalles de sus vidas profesionales, cuando se rompe la confianza por una violación de la confidencialidad y falta de transparencia y cuando la denuncia del acoso aumenta la vulnerabilidad a las represalias (Ahmed 2019; ASWG 2019:18; CSAT-US 2014:17; Wright 2003:230).

En tercer lugar, las acciones individuales y grupales pueden promover entornos de curación y recuperación.

Las respuestas basadas en evidencia que apoyan en lugar de re-traumatizar a les sobrevivientes y sus aliades incluyen: 
(1) Empoderar a les sobrevivientes y otres miembros vulnerables de nuestra comunidad profesional como arqueólogues exitoses que han enfrentado los efectos inmediatos y duraderos del trauma y el riesgo, con creatividad, autoconservación y determinación (CSAT-US 2014:3).

(2) Afirmar un compromiso fundamental para promover la seguridad física y emocional en la arqueología: "Crear seguridad no se trata de hacerlo bien todo el tiempo; se trata de la coherencia y la franqueza con que se manejan las situaciones . . . La comunicación honesta y compasiva que transmite la sensación de manejar la situación juntos genera seguridad" (CSAT-US 2014:20).

(3) Generar relaciones de confianza a través de procedimientos coherentes y transparentes relacionados con la denuncia y la adjudicación de denuncias de acoso (Johnson et al. 2018:6). En particular, es esencial que se respeten la autodeterminación, la privacidad y el derecho a desvincularse de les sobrevivientes.

(4) Desarrollar e implementar programas en colaboración con arqueólogues que son sobrevivientes de acoso, porque "les sobrevivientes son les expertes en lo que es mejor para elles" (NSACRSP/NSVRC 2017:9, 24).

(5) Desarrollar la competencia cultural para asegurar que los programas contra el acoso apoyen la inclusión y apunten a evitar el refuerzo de estereotipos dañinos, así como a colaborar con organizaciones no arqueológicas y personas expertas para mejorar los recursos existentes.

De esta manera, los enfoques basados en el trauma amplían la efectividad del modelo socioambiental al reconocer que la experiencia, el conocimiento y la creatividad de les sobrevivientes son recursos esenciales para abordar el acoso. El valor de un enfoque socioambiental y basado en el trauma se demostró en 2019, cuando el profesor emérito David Yesner se registró en la reunión anual de la Society for American Archaeology (SAA) en Albuquerque, New Mexico. Yesner había sido expulsado de la University of Alaska Anchorage debido a denuncias de conducta sexual inapropiada presentadas por nueve mujeres. Tres de las denunciantes estaban presentes en la conferencia.
Inicialmente, la SAA no dio mayor importancia a la asistencia de Yesner; sin embargo, su actitud de "seguir como si nada" terminó por re-traumatizar a las víctimas, creando un contexto de "gaslighting" (un modismo inglés que se refiere a una forma de abuso psicológico basado en hacer dudar a la víctima de su memoria o percepción del abuso) y de "culpar a la víctima" (ASWG 2019:17). Un tuit de la SAA reprendió a la University of Alaska Anchorage por no notificar a la SAA sobre la historia de Yesner. Les líderes de la SAA también bloquearon las cuentas de Twitter de les miembros que criticaban la manera en que la organización manejó la situación. Una de las sobrevivientes escribió en forma de protesta:

@SAAorg eligió ponerse del lado de un depredador sexual que ha sido investigado en forma privada y donde nuestros nueve testimonios fueron considerados creíbles. Tres de nosotras estamos aquí [SAA]. Decidieron que un anciano enfermo jubilado era más importante que tres mujeres jóvenes que recién comienzan y estamos enojadas. No me iré en silencio. \#saa2019 [Norma Johnson, citada en Rivera 2019].

Les sobrevivientes y sus aliades respondieron a la presencia de Yesner y a las respuestas de la SAA a través de intervenciones en varios niveles. Reportaron sus inquietudes a través de canales formales y redes sociales (nivel individual). Organizaron un sistema de apoyo para ayudar a les asistentes vulnerables a moverse con seguridad por el lugar, organizaron grupos de apoyo dirigidos por pares para les asistentes re-traumatizades, y se comunicaron con otres asistentes a través de las redes sociales, carteles impresos y anuncios en las sesiones de la conferencia (nivel de relación). También lanzaron una campaña con una carta pública (nivel organizacional), reclutaron declaraciones de apoyo de líderes de grupos de interés de la SAA y otros grupos arqueológicos o sociedades (nivel comunitario), y colaboraron con periodistas para generar conciencia pública sobre la situación (nivel social). Después de la conferencia, las sobrevivientes y aliades tomaron aún más acciones en todos estos niveles (ASWG 2019; Blackmore y Rutecki 2019; Collective Change 2019; Flaherty 
2019; Grens 2019; Hays-Gilpin et al. 2019; Killgrove 2019; Klembara y Markert 2019; Quinlan 2019; Rivera 2019; Wade 2019).

Estas intervenciones en varios niveles, alineadas al enfoque basado en el trauma, presionaron con éxito a les líderes de la SAA para que cambiaran de tono, restablecieran las cuentas de Twitter bloqueadas, emitieran disculpas formales, reembolsaran las tarifas de la conferencia pagadas por las víctimas de Yesner y reconocieran las graves deficiencias en las políticas y procedimientos de la SAA. También en respuesta a la presión de les sobrevivientes y sus aliades, en mayo de 2019, la SAA formó un Task Force on Sexual and Anti-Harassment Policies and Procedures. Al año siguiente, autorizó dos comités permanentes: (1) el Meeting Safety Committee, encargado de organizar medidas proactivas para apoyar la seguridad de les asistentes a la conferencia, y (2) el Findings Verification Committee, que responderá a los informes en caso de que algune miembro de la SAA sea declarade responsable de acoso (Hays-Gilpin et al. 2019:9; ver también SAA 2020a). Más allá de la SAA, otras organizaciones de la arqueología también se inspiraron para desarrollar políticas y procedimientos más efectivos como resultado de estas intervenciones, expandiendo el impacto de las acciones dirigidas por sobrevivientes a nivel de la comunidad.

\section{Intervenciones para atacar las culturas de acoso en la arqueología}

Primero, ¿qué has hecho en relación con las violencias físicas y estructurales generalizadas y endémicas que ocurren en tu disciplina? ... En segundo lugar, ¿qué vas a hacer ahora que tus amigues, colegas y estudiantes han expresado tan claramente sus preocupaciones, heridas y violaciones? ¿Cómo te curas? ¿Cómo responsabilizas a las personas o a ti misme? ¿Cómo incitas al cambio? ¿Cuál es tu camino (o el camino colectivo de nuestra disciplina) hacia la redención? [Collective Change 2019:13].

\section{Nivel social: Definición del acoso como mala conducta científica y profesional}

El acoso en la arqueología y en campos relacionados con STEMM (ciencia, tecnología, ingeniería, matemáticas y medicina) es tratado frecuentemente como un asunto interpersonal. Desde mediados de la década de 2010, legisladores, arqueólogues de agencias y sociedades profesionales se han involucrado en una amplia coalición para definir el acoso como una mala conducta científica y profesional, porque

daña, interfiere o sabotea la actividad y las carreras científicas. El acoso y la agresión sexual crean un entorno hostil que reduce la calidad, la integridad y el ritmo del avance de la ciencia al marginar a las personas y las comunidades. También daña la productividad y el avance profesional, e impide el intercambio saludable de ideas [Southeastern Archaeological Conference (SEAC) 2018; véase también Clancy et al. 2014].

La clasificación del acoso como una mala conducta científica y profesional establece que es tan perjudicial para la confianza pública como el plagio, la falsificación de datos, el saqueo y tráfico de antigüiedades, las citaciones coercitivas y el uso fraudulento de fondos de investigación. Presentada por primera vez en la United States House of Representatives en 2016, la ley Combating Sexual Harassment in Science Act, si se aprueba, elevaría el acoso al nivel de conducta poco ética para fines federales, y requeriría que cualquier universidad que reciba subvenciones informe públicamente todos los hallazgos fundamentados de agresión y acoso sexual por parte de instructores e investigadores principales (Johnson 2019; Speier 2016). Aunque el proyecto de ley aún no se ha sometido a votación, la National Science Foundation (NSF) ya ha emitido regulaciones que reflejan la legislación propuesta (Córdova 2018), al igual que algunas sociedades arqueológicas.

Al definir el acoso como mala conducta científica, les arqueólogues también pueden tomar medidas para asegurarse de que no estamos habilitando a les acosadores a través de nuestras relaciones profesionales: "Cada artículo que publican, charla que dan y conferencia a la que asisten aumenta la influencia de la que han abusado" (Wood 2015:487). Como Bhalla (2018) sugiere, "hay que debilitar el estatus que continúan disfrutando algunes acosadores en serie. No colabore con elles. No les invite a reuniones, 
a dar seminarios, etc. No les invite a ser IP [investigadores principales] en una beca de capacitación ni a participar en un programa de posgrado." En un ensayo titulado "What to Do with the Predator in Your Bibliography", Souleles (2020) recomienda medidas específicas para evitar elevar el estatus de les acosadores a través de citaciones: reconocer el abuso que ocurrió durante la investigación citada, considerar alternativas a citar a les abusadores y trabajar para mover el campo más allá de las contribuciones de les abusadores.

\section{Niveles de comunidad y organización}

Una organización es cualquier entidad que conecta personas y recursos en relación con una meta o misión. En consecuencia, una organización arqueológica puede ser tan pequeña como un club de arqueología universitario de cinco estudiantes o tan grande como una organización profesional como la SAA (con alrededor de 7.500 miembros en 2020; SAA 2020b). Las organizaciones pueden estar en red horizontal (como en las conexiones entre asociaciones profesionales) o anidadas verticalmente (como un laboratorio en un centro de investigación, un departamento en una universidad o la oficina regional de una empresa). Estas relaciones interorganizacionales constituyen el nivel comunitario del modelo socioambiental.

Las organizaciones generan los contextos en los que se produce el acoso laboral y educativo. Las organizaciones también determinan el grado en que les acosadores serán formalmente responsables de sus acciones, y qué recursos, si los hay, estarán disponibles para apoyar a les sobrevivientes y protegerles de represalias. En consecuencia, "el indicador más potente del acoso sexual es el clima organizacional: el grado en que aquelles en la organización perciben que el acoso sexual es o no tolerado" (Johnson et al. 2018:x).

La promoción tanto de les líderes como de les miembros es fundamental para transformar la cultura organizacional y adoptar las mejores prácticas para prevenir el acoso, apoyar a les sobrevivientes y responsabilizar a les acosadores por sus acciones. El resto de esta sección describe los enfoques basados en evidencia que sirven como pautas para las organizaciones arqueológicas que buscan abordar el acoso, con atención especial al final de la sección a los contextos de investigación de campo arqueológico.

Empoderamiento de sobrevivientes y miembros vulnerables. Les sobrevivientes del acoso y otres miembros vulnerables sabrán cuáles son los problemas o quiénes son, por qué persiste el acoso en la organización y qué se puede hacer para detenerlo. Las organizaciones pueden patrocinar foros y audiencias de escucha para unir la experiencia individual al nivel de la organización y hacer visibles las deficiencias de sus políticas y procedimientos (Collective Change 2019:13).

Encuestas climáticas. Son necesarias encuestas periódicas para identificar el alcance y el impacto del acoso, así como para evaluar si las intervenciones a nivel de la organización son efectivas para reducir el acoso, mejorar los reportes y apoyar a les sobrevivientes.

Códigos de conducta con recursos y sanciones. A diferencia de las declaraciones de política pública, los códigos de conducta organizacionales tienen un impacto claro y demostrable en la reducción del acoso y la agresión sexual porque especifican claramente las consecuencias de la mala conducta (Bradford y Crema 2020; Clancy et al. 2014; Nelson et al. 2017). Los códigos de conducta deben aplicarse no sólo a les miembros habituales de una organización, sino también a les visitantes y subcontratistas. El código de conducta y las comunicaciones relacionadas deben "transmitir que denunciar el acoso sexual es una acción honorable y valiente" (Johnson et al. 2018:7) que mantiene la integridad de la organización. Los códigos de conducta también deben indicar claramente que no se tolerarán las represalias contra quienes denuncien el acoso.

Los códigos de conducta efectivos tienen "mecanismos para responsabilizar a les miembros de la comunidad y a les líderes, en todos los niveles, de cumplir con las expectativas culturales de comportamiento" (Collective Change 2019:14; véase también Aurora y Gardiner 2019). Con demasiada frecuencia, las acciones que toman las organizaciones contra les acosadores parecen recompensas:

Incluso en los casos donde los reportes de acoso brindan resultados, las víctimas 
pueden ver a sus agresores "castigades" con permisos sabáticos, sin obligaciones de enseñanza, y sin ningún obstáculo para continuar con su investigación, avanzar profesionalmente en otra universidad, o gozar de una jubilación temprana con plenos beneficios [Marín-Spiotta et al. 2016].

Las verdaderas sanciones son apropiadas en relación con la gravedad de la violación del código de conducta y podrían incluir la restricción de actividades en la organización; suspender o rescindir la membresía, el empleo o la inscripción; rescindir premios u honores; $\mathrm{y}$ enmendar o retractar publicaciones e informes de investigación para señalar claramente la mala conducta que ocurrió durante la actividad de investigación. Las organizaciones también pueden exigir a les acosadores encontrades culpables que completen programas de capacitación como parte de un plan de reingreso, pero la capacitación en sí nunca debe utilizarse como sustituto de las sanciones apropiadas.

Procedimientos claros y efectivos para la presentación de informes y adjudicación. Con frecuencia, les acosadores están en una posición de poder sobre sus víctimas, por lo que los reportes que pasan por toda la cadena de mando aumentan la vulnerabilidad de las personas. La raza, la clase, la nacionalidad, el estado de inmigración, la capacidad física y las identidades sexuales y de género complican aún más la posibilidad de las víctimas para reportar de forma segura sus experiencias. En consecuencia, es importante crear múltiples puntos de contacto, incluyendo a personas con diferentes identidades y algunas que están fuera del organigrama, como une ombudsperson o une funcionarie externe designade (Heath-Stout 2019:336). La información sobre cómo denunciar el acoso debe ser claramente visible para todos les miembros, especialmente para les estudiantes y les profesionales principiantes. Estas medidas ayudan a garantizar que cualquier arqueólogue que haya sido objeto de acoso pueda "sentirse cómode reportando a una entidad preocupada y empoderada" (Radde 2018:251).

Debido a que las víctimas de acoso a menudo se resisten a denunciar, las organizaciones también pueden desarrollar procedimientos de divulgación a través de los cuales una víctima o espectadore puede alertar anónimamente sobre una violación del código de conducta sin desencadenar una denuncia y una investigación completa. Esto proporciona otra forma en que la organización pueda tomar acciones para proteger a sus miembros. Junto con las vías formales para la difusión, las organizaciones pueden estar atentas a las divulgaciones informales realizadas a través de publicaciones en redes sociales, sitios web u otros foros.

La formación y la educación se centran en el comportamiento, no en las creencias - esto debe ser obligatorio y recurrente. Debido a que las actitudes y comportamientos que contribuyen al acoso a menudo se mantienen a nivel social, se necesita capacitación continua dentro de cada organización arqueológica para ayudar a les miembros a reconocer, intervenir y reportar el comportamiento de acoso. Se debe exigir educación a todes les miembros al entrar a la organización, con capacitación especializada para arqueólogues junior, titulares de cargos, instructores, supervisores y arqueólogues en puestos de cara al público. La capacitación a nivel de organización es más efectiva cuando se enfoca en "cambiar el comportamiento, no en cambiar las creencias" y "en comunicar claramente las expectativas de comportamiento [y] especificar las consecuencias por no cumplir con estas expectativas" (Johnson et al. 2018:5). La capacitación que "combina los esfuerzos contra el acoso con los programas de promoción del civismo" proporciona a les miembros habilidades para "interrumpir e intervenir cuando se produce un comportamiento inapropiado" (Johnson et al. 2018:5).

Mitigar el efecto de les gatekeepers. Las organizaciones arqueológicas pueden combatir las relaciones jerárquicas creadas por les gatekeepers reestructurando sus operaciones de modo que ningún individuo tenga poder directo sobre otres arqueólogues. Por ejemplo, los programas educativos pueden instituir la coadministración y las empresas pueden cambiar a un modelo de supervisión basada en equipos. En lugar de conferir las decisiones sobre permisos de investigación o acceso a colecciones a una sola persona. Una agencia o museo podría establecer un comité de personal para revisar las solicitudes. Ya sea 
que la autoridad para tomar decisiones esté en manos de una sola persona o sea compartida, los procedimientos y criterios transparentes, con procesos de apelación, son importantes para disuadir a les "abusadores de poder" de sacar ventajas de la autoridad de su puesto.

Coordinación a nivel comunitario. El intercambio de recursos vertical y horizontal entre organizaciones ayuda a reducir los costos y a compartir la experiencia práctica, al mismo tiempo que establece estándares consistentes en toda la disciplina. Por ejemplo, SEAC (2019) ha desarrollado plantillas de códigos de conducta para reuniones profesionales y proyectos de campo, así como folletos y módulos de capacitación que se pueden adaptar para proyectos, divisiones, laboratorios y aulas específicas. En el Reino Unido, el Chartered Institute for Archaeologists (2020) ha unido fuerzas con una organización benéfica para proporcionar a les arqueólogues de CRM acceso a recursos de capacitación, canales de información confidenciales y abogades cualificades.

Las organizaciones también pueden aprovechar sus recursos para apoyar un cambio positivo en otras organizaciones. Por ejemplo, durante casi 40 años, la American Anthropological Association (AAA) ha utilizado su papel como difusora de oportunidades para incentivar la equidad y la inclusión (Levine 1994:23). Desde 2018, la AAA exige que todas las escuelas de campo y otras experiencias de investigación anunciadas "pongan a disposición un código de conducta que prohíba la agresión y el acoso sexual", y que den fe de que existen "mecanismos de denuncia apropiados para aquelles que sufren o presencian acoso o agresión sexual" (AAA 2020). En 2019 el Register of Professional Archaeologists (RPA), la SAA y el Archaeological Institute of America (AIA) adoptaron políticas similares. $^{2}$

Proyectos de campo como organizaciones especiales en la arqueología. Debido a que los proyectos de campo son "experiencias altamente sociales en las que las personas tienen que vivir juntas y cooperar como parte de un grupo" (Moser 2007:246-247), éstos plantean desafíos específicos para prevenir y denunciar el acoso. Los recursos limitados y el tiempo disponible para la investigación de campo pueden llevar a "priorizar la generación de datos [que] puede contribuir a descuidar, de forma benigna o no, la dinámica del equipo, de manera que puede ocurrir alienación, acoso y abuso" (Clancy et al. 2014:1). Debido a que la experiencia de campo es necesaria para la capacitación arqueológica, y que a menudo implica costosos arreglos financieros y logísticos (Heath-Stout y Hannigan 2020), les participantes pueden tener incentivos adicionales para soportar condiciones adversas en lugar de abandonar el proyecto o denunciar el acoso.

A pesar de estos desafíos, prevenir el acoso en los proyectos de campo arqueológico es una prioridad absoluta si la arqueología, como disciplina, quiere ir más allá de una cultura generalizada del acoso. Como afirman Colaninno y colaboradores,

Les aspirantes a arqueólogues pueden sufrir su primera experiencia de acoso sexual, agresión y violencia como estudiantes de pregrado o posgrado matriculades en una escuela de campo . . . También puede ser la primera vez que se ignoran o pasan por alto intencionalmente las preocupaciones y los informes de acoso y agresión de un estudiante. Además, les futures perpetradores pueden ver que el acoso y la agresión sexual son componentes normales del trabajo de campo arqueológico, perpetuando así esta cultura de tolerancia [Colaninno et al. 2020:112, 114].

En los últimos dos años, se han publicado varias guías (Colaninno et al. 2020; Hanes y Walters 2020; SEAC 2019; Walter y Bergstrom 2020) y un programa de capacitación sin fines de lucro (Fieldwork Initiative 2020) para orientar a les directores de proyectos de campo y a les participantes en la prevención del acoso y en el apoyo al bienestar de todes. Estas guías, junto con los hallazgos de estudios de investigación centrados en contextos de campo (Bradford y Crema 2020; Clancy et al. 2014; Heath-Stout 2019; Nelson et al. 2017; Radde 2018), enfatizan que los códigos de conducta que prohíben el acoso - con claros mecanismos de aplicación - son el factor más importante que influye en el acoso en los contextos de investigación de campo. Los códigos de conducta son más efectivos cuando se 
distribuyen antes del proyecto y se refuerzan mediante capacitación en persona (Bradford y Crema 2020). Es particularmente crítico tener una lista de contactos para el reporte inmediato y para que la intervención vaya más allá de le investigadore principal y les directores de proyecto (AIA 2019:1-3; Heath-Stout 2019:336; Radde 2018:250). Bradford y Crema (2020) encontraron que la frecuencia del acoso en los proyectos de campo estaba correlacionada con la duración del proyecto. En consecuencia, los proyectos más largos deben incluir cursos de refuerzo. Colaninno y colaboradores (2020:117) recomiendan encuestas confidenciales semanales de todes les miembros del proyecto, así como reflexiones grupales, para que les líderes del proyecto puedan abordar fácilmente los problemas a medida que surgen.

Los códigos de conducta para proyectos de arqueología que implican residencia continua deben abordar el comportamiento tanto personal como profesional. Las bromas discriminatorias crean un ambiente de "hostilidad, agresión e intolerancia hacia la diferencia" (encuestade anónime, citado en Radde 2018:246) y deben prohibirse en todo momento. La fraternización es especialmente peligrosa durante el trabajo de campo, y la AIA (2019:2) recomienda la prohibición absoluta de "las relaciones entre cualquier persona en un rol de supervisión y cualquier otra persona que desempeñe una función bajo esa supervisión". Se necesitan reglas claras sobre el consumo de alcohol para prevenir el desarrollo de una "cultura de fiesta" que permita a les acosadores eludir la responsabilidad (Heath-Stout 2019; véase también Hodgetts et al. 2020). Colaninno y colaboradores (2020:118) recomiendan eso debido al "vínculo conocido entre el uso de sustancias y el comportamiento sexualmente inapropiado . . . la disciplina debe llamar a les directores para implementar escuelas de campo libres de alcohol y drogas para los programas residenciales". Los códigos de conducta de los proyectos de campo también deben enfatizar que las decisiones de les participantes en torno a las drogas o el alcohol de ninguna manera excusan las acciones de les perpetradores o culpabilizan a las víctimas.

El transporte, la vivienda y la comunicación plantean desafíos específicos para garantizar el bienestar de les participantes en los proyectos de arqueología residencial. Debido a que los sitios de campo a menudo son remotos, "les estudiantes tienen el potencial de sentirse atrapados con su acosadore/abusadore, lo que puede fomentar un miedo más inmediato al daño corporal y la ansiedad mental" (Hanes y Walters 2020). El sentimiento de lejanía y de estar aislade de las redes sociales preexistentes puede ocurrir, sea el proyecto nacional o internacional. Es fundamental asegurar que les participantes puedan comunicarse con personas fuera del sitio de campo, especialmente con servicios confidenciales y oficinas de informes, al igual que garantizar que todes les participantes tengan acceso a transporte seguro en caso de que deseen abandonar el proyecto. Proporcionar información anticipada sobre los arreglos de vivienda, respetar las identidades de género de les participantes y evitar el uso de "supuestos roles de género heterosexuales" al asignar viviendas en el campo contribuyen a condiciones más seguras (Blackmore et al. 2016:22; ver también Dylla et al. 2016; HeathStout 2019:336). Al planificar proyectos de campo, considere la posibilidad de que, si se hace una denuncia de acoso o agresión sexual, puede ser necesario proporcionar a la persona que reporta y a le acusade espacios de vida separados y seguros durante y después de la investigación, reembolsos de cuotas o licencia pagada, y transporte seguro a casa (Hanes y Walters 2020:9).

\section{Nivel relacional}

Transformar nuestra cultura disciplinaria también requiere acciones positivas en nuestras relaciones profesionales. Una de las intervenciones más importantes es hablar abierta y francamente con otres arqueólogues sobre el acoso. Podemos enfatizar nuestra admiración y apoyo a les sobrevivientes y les testigos que denuncian el acoso. De esta forma, "se difama el mal comportamiento y se apoya a las víctimas para que actúen si así lo desean" (Radde 2018:250). También es importante ampliar la autonomía y la agencia de les sobrevivientes, no sólo apoyando su lucha de elles mismes y otres, sino también respetando sus necesidades de privacidad y desconexión.

Debido a que la cultura arqueológica ha valorado históricamente la informalidad en las relaciones profesionales (Leighton 2020; Moser 
2007), les arqueólogues podemos sentirnos incómodes al reconocer que ocupamos roles de supervisión y control. Cualquiera que sea nuestra posición, reconocer las diferencias de poder en nuestras relaciones profesionales es esencial para romper las culturas de acoso en la arqueología. Es especialmente importante recordar que les subordinades no pueden dar su consentimiento libremente a las propuestas personales e íntimas de les instructores, supervisores y gatekeepers. Les supervisores y gatekeepers pueden, en ocasiones, malinterpretar el comportamiento apropiado de una persona subordinada (respeto profesional, atención y deferencia) como interés personal. Independientemente de las acciones de le subordinade, siempre es responsabilidad de les supervisores mantener límites profesionales claros.

Un enfoque basado en el trauma nos ayuda a comprender que los límites claros en nuestras relaciones profesionales promueven una cultura disciplinaria inclusiva y de apoyo. Por ejemplo, cuando era estudiante y arqueóloga principiante, era muy común que me invitaran a las casas de les supervisores o a bares para reuniones personales fuera del horario laboral. A medida que ascendía de rango, envié invitaciones similares al personal y a les estudiantes. Desafortunadamente, esta práctica común ha dado cobertura a les acosadores que usan invitaciones "amistosas" para encontrar a posibles víctimas. He aquí un ejemplo:

De regreso a la ciudad, Amanda me pidió que la acompañara a su casa. En su sala no estaba segura de sus intenciones ... Ella fue mi consejera. No podía permitirme el lujo de pensar mal, de actuar mal, así que me quedé paralizada [She 2000:170].

La adopción de un enfoque más formal en las interacciones profesionales no tiene por qué afectar negativamente a la colegialidad que muches arqueólogues valoran; de hecho, puede mejorar nuestra cultura disciplinaria al fomentar un entorno más inclusivo.

El nivel relacional es especialmente importante para apoyar a les colegas jóvenes y compañeres que son objeto de acoso. Muches arqueólogues de alto nivel, incluyéndome a mí, fueron entrenades para soportar más que enfrentar el acoso. Al adoptar una perspectiva basada en el trauma, podemos responder a las revelaciones de acoso, empoderando a les supervivientes para que tomen las acciones que consideren más adecuadas para su propio bienestar.

\section{Nivel individual}

Las intervenciones en los niveles relacional, organizacional, comunitario y social se enfocan en el comportamiento más que en las creencias. A nivel individual, un enfoque centrado en valores genera principios básicos que pueden guiar la conducta individual (Hays-Gilpin et al. 2019:9; Nelson et al. 2017:718). A menudo, esto comienza con la autorreflexión: ¿Qué significa para mí ser arqueólogue? ¿Qué es lo que valoro de ser parte de esta comunidad profesional? ¿Cuáles son los valores que aporto a la arqueología dados mis antecedentes personales y las comunidades no profesionales a las que pertenezco?

Conectarse con los valores fundamentales ayuda a les arqueólogues a navegar en situaciones en las que nuestro propio interés puede entrar en conflicto con la promoción de un entorno libre de acoso. Por ejemplo, en un proyecto que dirigí, tres participantes me informaron que se sentían incómodes debido a las miradas y comentarios de un gatekeeper que visitaba con frecuencia el proyecto. También me había sentido incómoda con el comportamiento de esta persona, pero temía que confrontar al gatekeeper pudiera poner el proyecto en peligro. Entonces, hice caso omiso de las quejas de les participantes y les dije que no veía ninguna evidencia de un problema real.

$\mathrm{Si}$, en cambio, hubiera seguido mis valores fundamentales, el compromiso con la honestidad y el derecho de tode arqueólogue a aprender y trabajar en un entorno seguro e inclusivo, habría respondido de manera diferente. Podría haber contactado a mentores para pedirles consejo, o haber reconocido el conflicto de intereses y buscar recursos externos que pudieran haber aportado perspectivas más imparciales, como por ejemplo otros recursos humanos o contactar a las oficinas del Title IX. O podría haber hablado directamente con el gatekeeper sobre cómo su comportamiento estaba afectando al equipo.

Cuando reflexiono sobre mi carrera, veo claramente que a menudo creía que yo era impotente para cambiar la cultura del acoso en la 
arqueología. Muches de nosotres nos hemos sentido incapaces de evitar que el acoso nos suceda a nosotres y a otres arqueólogues. Muches denunciamos valientemente el acoso y luego descubrimos que no se hizo nada para protegernos o para responsabilizar a nuestres perpetradores. Con el tiempo, estas experiencias pueden erosionar nuestro sentido de agencia.

Un enfoque basado en el trauma nos ayuda a comprender que nuestros sentimientos iniciales de impotencia son respuestas normales a situaciones anormales. Estas y otras estrategias de afrontamiento pueden habernos ayudado a sobrevivir en circunstancias difíciles y permitido perseverar en la arqueología. Reflexionar sobre nuestros valores fundamentales nos permite reconocer nuestra creatividad y resiliencia y aplicarlos para interrumpir el acoso en la arqueología.

Como arqueólogues, podemos empoderarnos individualmente, preparándonos con anticipación para abordar el acoso si ocurre. Una lista de verificación personal podría incluir: (1) saber cómo comunicarse con recursos confidenciales para obtener apoyo; (2) aprender sobre los canales para la presentación de reportes formales; (3) establecer un plan de comunicación con amigues o colegas de confianza fuera del lugar de trabajo, programa educativo o sitio de campo; (4) solicitar códigos de conducta e información sobre recursos de comunicación, transporte y (para proyectos residenciales) alojamiento; (5) identificar los límites relacionados con la información personal, el consumo de alcohol y drogas, y las interacciones sociales fuera del horario de trabajo o del proyecto.

Les arqueólogues pueden amplificar aún más estas fortalezas buscando capacitación y tutoría "en las habilidades interpersonales de manejo de conflictos, negociación y resolución que les permitiría enfrentar de manera informal y formal los problemas de personal a medida que surgen y antes de que puedan escalar" (Clancy et al. 2014:1). Como espectadores, podemos intervenir si somos testigos de acoso, dirigiéndonos directamente les acosadores: "¡Alto, retroceda!" (Bhalla 2018), “¡Eso no está bien!” (Heath-Stout 2019), "Dejemos ese tipo de charlas" (Lavery 2014), "[Ese comportamiento] no está respaldado por nuestro código de conducta; tienes que parar" (SAA 2015). Comentarios como estos interrumpen el acoso al redirigir la atención de les acosadores hacia nuestra interrupción y lejos de su objetivo. También pueden ayudar a cambiar la experiencia de la víctima, "de sentimientos de impotencia y aislamiento a sentimientos de apoyo y justificación" (Lavery 2014).

\section{Conclusión}

El acoso ha moldeado nuestra disciplina a un nivel fundamental, afectando a quienes pueden practicar la arqueología, a cómo se entrena a les arqueólogues, a los temas de investigación, y a cómo se interpretan, publican y citan los datos arqueológicos. Aunque los estudios realizados en los últimos diez años han documentado la magnitud del problema, todavía queda mucho trabajo por hacer. Las experiencias de arqueólogues no binarios, arqueólogues de color, arqueólogues LGBTQIA+ y arqueólogues con discapacidades están muy poco estudiadas. Se necesita una investigación específica para asegurar que las experiencias de arqueólogues ya marginades no se descuiden más.

En los últimos cinco años, muchas organizaciones arqueológicas han iniciado nuevas políticas y procedimientos destinados a prevenir el acoso. Es más posible que estas intervenciones tengan éxito cuando se desarrollan en colaboración con sobrevivientes y otres arqueólogues vulnerables, y cuando aprovechan los recursos organizacionales para apoyar el cambio en múltiples niveles del modelo socioambiental. Necesitamos ver esto como un proceso continuo: se requieren encuestas climáticas periódicas junto con mecanismos de retroalimentación continua para evaluar la efectividad de las intervenciones específicas.

Los modelos socioambientales y los enfoques basados en el trauma son transformadores porque nos ayudan a ver que todes les arqueólogues tienen la capacidad de interrumpir las culturas de acoso. La mayoría de les arqueólogues valoran profundamente nuestra disciplina compartida y nuestras relaciones. Estos valores fundamentales son nuestra fuerza colectiva. Juntes, podemos interrumpir los ciclos intergeneracionales de acoso y crear una arqueología más inclusiva.

\section{Coda: ¿Dónde ir por ayuda?}

Puede ser abrumador conocer la omnipresencia del acoso en la arqueología. Para les sobrevivientes 
y les testigos puede resultar valioso e inquietante saber que nuestras experiencias negativas son parte de un patrón amplio de toda la disciplina. Sea usted sobreviviente o testigo de acoso o agresión sexual, o que conozca a alguien que haya sufrido o sido testigo de ello, no está sole. Hay ayuda disponible. Si no está seguro por dónde empezar, en los Estados Unidos existe la Rape, Abuse \& Incest National Network (RAINN), que brinda apoyo gratuito y confidencial a les sobrevivientes y a quienes se preocupan por elles. La ayuda está disponible las 24 horas del día, los siete días de la semana por teléfono (800-656-4673) y por chat en línea: https:// www.rainn.org/. En español, llame al 800-656-4673 a la Línea de Ayuda Nacional Online de Asalto Sexual o comuníquese a través de la opción "Chat Ahora": https://www.rainn.org/es.

Agradecimientos. Este artículo está dedicado a todes les sobrevivientes de acoso en la arqueología. Estoy especialmente agradecida a aquelles que han realizado investigaciones sobre el acoso y a les activistas y defensores a lo largo de la historia de la arqueología. Comencé a escribir esta serie de artículos durante mi participación en comités y programas contra el acoso en mi lugar de trabajo. Mi agradecimiento a muches colegas, estudiantes, personal y administradores (demasiades para nombrarles a todes aquí) que compartieron sus conocimientos y experiencias, y a Danielle J. Bradford, Kathryn Clancy, Deb Cohler, Carol Colaninno, Kathy Coll, Maura Finkelstein, Kelley HaysGilpin, Mark Hauser, Laura Heath-Stout, Lochlann Jain, Caren Kaplan, Kristina Killgrove, Hugh D. Radde, Doug Rocks-Macqueen, Maureen S. Meyers, Sarah Rowe, Dawn Rutecki, Megan Thies-Sauder, Sasja van der VaartVerschoof, y a siete lectores anónimos que revisaron la versión publicada en American Antiquity y que proporcionaron comentarios reflexivos sobre borradores anteriores. Un agradecimiento especial a Lynn Gamble por su apoyo y orientación editorial, Laura Daly por la corrección de estilo, y Katie Johnson-Noggle por el diseño gráfico.

Mi agradecimiento a Grace Alexandrino Ocaña, Francesca Giulietta Fernandini Parodi, Alberto Quintero Soriano, a les editores de Latin American Antiquity Julia Hendon y Calogero Santoro, a María Cecilia Sanhueza, y a varias personas anónimas por la traducción al español del manuscrito. El Director's Fund del Stanford Archaeology Center Director's Fund financió generosamente Gold Open Access para este artículo.

Conflicto de intereses. La autora no tiene conocimiento de ningún interés financiero o afiliación que plantee un conflicto de intereses con el contenido de este artículo.

Declaración de disponibilidad de datos. Este estudio se basa en el análisis de investigaciones publicadas, relatos de sobrevivientes, informes de noticias y recursos en línea. Todos los datos están disponibles a través de las fuentes enumeradas en la sección Referencias citadas.

\section{Notas}

1. Lesbianas, gays, bisexuales, transgénero/transexuales/trans, queer, intersexuales, asexuales, más los no binarios, y muchas otras personas cuyo género y/o sexualidad real o percibida no se ajusta a las normas sancionadas, junto con les aliades de LGBTQIA+ individuos y comunidades.

2. A principios de la década de 2010 , varias organizaciones de arqueología alentaron o exigieron que las escuelas de campo tuvieran políticas de acoso sexual. La AAA fue la primera en exigir códigos de conducta con sanciones obligatorias.

\section{Referencias citadas}

Ahmed, Sarah

2019 Why Complain? Feministkilljoys (blog), el 22 de julio. https://feministkilljoys.com/2019/07/22/why-complain/, accedido el 29 de enero de 2020.

American Anthropological Association (AAA)

2020 List in the AnthroGuide. Documento electrónico, https://guide.americananthro.org/24875/List-in-theAnthroGuide, accedido el 8 de septiembre de 2020.

Archaeological Institute of America (AIA)

2019 Archaeological Institute of America Statement on Sexual Harassment and Assault and Guidelines for Archaeological Field Projects. Documento electrónico, https://www.archaeological.org/wp-content/uploads/ 2019/05/AIA-Statement-on-Sexual-Harassment-andAssault-and-Guidelines-on-Archaeological-FieldProjects.pdf, accedido el 4 de febrero de 2020.

Aurora, Valerie y Mary Gardiner

2019 How to Respond to Code of Conduct Reports. Documento electrónico, https://frameshiftconsulting.com/ code-of-conduct-book/, accedido el 28 de noviembre de 2020.

Awesome Small Working Group (ASWG)

2019 Take Back the SAA: Member-Led Organizing and Action in the Wake of \#SAA2019. SAA Archaeological Record 19(4):16-21.

Bhalla, Needhi

2018 A Beginner's Guide for Addressing Sexual Harassment in Academia. Edge for Scholars (blog). https://edgeforscholars.org/a-protocol-for-addressingsexual-harassment-and-assault/, accedido el 3 de diciembre de 2019 .

Bikales, James S.

2020 Protected by Decades-Old Power Structures, Three Renowned Harvard Anthropologists Face Allegations of Sexual Misconduct. Harvard Crimson, el 29 de mayo. Documento electrónico, https://www.thecrimson. com/article/2020/5/29/harvard-anthropology-gender-issues/, accedido el 17 de agosto de 2020.

Blackmore, Chelsea, Leslie Drane, Richard Baldwin y David Ellis

2016 Queering Fieldwork: Difference and Identity in Archaeological Practice. SAA Archaeological Record 16(1):18-23.

Blackmore, Chelsea y Dawn M. Rutecki

2019 Open Letter to the Society of American Archaeology President, Board of Directors, and Executive Director. From co-organizers of the Queer Archaeology 
Interest Group, el 19 de abril. Documento electrónico, https://docs.google.com/document/d/10GLSYL10PN1j ka18_S9V5xh_pk6F7CN6oqrfPve5WEo/edit?fbclid= IwAR341R9gqK4CMzerdXrbnfygZYqajDKNljkEtSXGmaf21HPYUIROz3Kg4, accedido el 22 de abril de 2019.

Bradford, Danielle J. y Enrico R. Crema

2020 Risk Factors for the Occurrence of Sexual Misconduct during Archaeological and Anthropological Fieldwork. Manuscrito en archivo, McDonald Institute for Archaeological Research, University of Cambridge.

Center for Substance Abuse Treatment (CSAT-US)

2014 Trauma-Informed Care in Behavioral Health Services. Treatment Improvement Protocol (TIP) Series 57. Substance Abuse and Mental Health Services Administration (US), Rockville, Maryland.

Centers for Disease Control and Prevention

2019 The Social-Ecological Model: A Framework for Prevention. Documento electrónico, https://www.cdc. gov/violenceprevention/publichealthissue/social-ecolo gicalmodel.html, accedido el 8 de enero de 2020.

Chartered Institute for Archaeologists

2020 Protect: Speak Up, Stop Harm. Documento electrónico, https://www.archaeologists.net/membership/pcaw, accedido el 27 de noviembre de 2020.

Clancy, Kathryn B. H., Robin G. Nelson, Julienne N. Rutherford y Katie Hinde

2014 Survey of Academic Field Experiences (SAFE): Trainees Report Harassment and Assault. PLOS ONE 9 (7):e102172. DOI:10.1371/journal.pone.0102172.

Colaninno, Carol E., Shawn P. Lambert, Emily L. Beahm y Carl G. Drexler

2020 Creating and Supporting a Harassment- and AssaultFree Field School. Advances in Archaeological Practice 8:111-122.

The Collective Change

2019 \#MeToo in Archaeology. SAA Archaeological Record 19(4):12-15.

Córdova, France A.

2018 Important Notice No. 144. Harassment: Important Notice to Presidents of Universities and Colleges and Heads of Other National Science Foundation Grantee Organizations. National Science Foundation, el 8 de febrero. Documento electrónico, https://www.nsf.gov/ pubs/issuances/in144.jsp, accedido el 10 de enero de 2020.

Coto-Sarmiento, María, Lara Delgado Anés, Lourdes López Martínez, Jesús Martín Alonso, Ana Pastor Pérez, Apen Ruíz y María Yubero Gómez

2018 Informe sobre el acoso sexual en arqueología (España). Zenodo. Documento electrónico, http://doi. org/10.5281/zenodo.3662763, accedido el 27 de febrero de 2020.

Crenshaw, Kimberlé

1991 Mapping the Margins: Intersectionality, Identity Politics, and Violence Against Women of Color. Stanford Law Review 43:1241-1299.

Dylla, Emily, Sheena A. Ketchum y Carol McDavid

2016 Listening More and Talking Less: On Being a Good Ally. SAA Archaeological Record 16(1):31-36.

Fieldwork Initiative

2020 The FISST Training. Documento electrónico, http:// fieldworkinitiative.org/the-fisst-training/, accedido el 5 de mayo de 2020.

Flaherty, Colleen

2019 Archaeology Group Faces Backlash over How It Handled Known Harasser's Attendance at Meeting.
Inside Higher Ed, el 15 de abril. https://www.insidehig hered.com/news/2019/04/15/archaeology-group-facesbacklash-over-how-it-handled-known-harassers-attendance, accedido el 3 de diciembre de 2019.

Geller, Pamela L.

2016 This Is Not a Manifesto: Archaeology and Feminism. En Meta-Philosophical Reflection on Feminist Philosophies of Science, editado por Maria Cristina Amoretti y Nicla Vassallo, pp. 151-170. Springer International, Cham, Suiza.

Grens, Kerry

2019 Archaeologists Ask Society for Harassment Policy Change. The Scientist, el 16 de abril. Documento electrónico, https://www.the-scientist.com/news-opinion/ archaeologists-ask-society-for-harassment-policy-change65745 ? fbclid=IwAR15Y3zpyH79BrsS0SdQVg8b cdU6nVVXgG4THM5bwQu_9vDsVRbT7YYIDg, accedido el 19 de noviembre de 2020

Hanes, Amy y Holly Walters

2020 A Long Journey Home: Supporting Students in the Field. Documento electrónico, https://metooanthro.org, accedido el 22 de septiembre de 2020.

Hays-Gilpin, Kelley, Meagan Thies-Sauder, Catherine Jalbert, Laura Heath-Stout, Heather Thakar, Kenneth Aitchison, Jon Driver, Kisha Supernant y Amber M. VanDerwarker 2019 Changing Our Professional Culture of Apathy and Creating Safety in Archaeology. SAA Archaeological Record 19(4):8-11.

Heath-Stout, Laura Ellen

2019 Diversity, Identity, and Oppression in the Production of Archaeological Knowledge. Tesis doctoral, Department of Anthropology, Boston University, Boston, Massachusetts.

Heath-Stout, Laura Ellen y Elizabeth M. Hannigan

2020 Affording Archaeology: How Field School Costs Promote Exclusivity. Advances in Archaeological Practice 8:123-133.

Hodgetts, Lisa, Kisha Supernant, Natasha Lyons y John R. Welch

2020 Broadening \#MeToo: Tracking Dynamics in Canadian Archaeology Through a Survey on Experiences Within the Discipline. Canadian Journal of Archaeo$\log y$ 44:20-47.

Jalbert, Catherine L.

2019 Archaeology in Canada: An Analysis of Demographics and Working Conditions in the Discipline. Tesis doctoral, Department of Archaeology, Memorial University of Newfoundland, St. John's, Canadá.

Johnson, Eddie Bernice

2019 Combating Sexual Harassment in Science Act of 2019, H.R. 36, 116th Cong. Documento electrónico, https://www.congress.gov/bill/116th-congress/house-bill/ 36, accedido el 28 de septiembre de 2020.

Johnson, Paula A., Sheila E. Widnall y Frazier F. Benya (editores)

2018 Sexual Harassment of Women: Climate, Culture, and Consequences in Academic Sciences, Engineering, and Medicine. Consensus Study Report of the National Academies of Sciences, Engineering, and Medicine. National Academies Press, Washington, DC.

Killgrove, Kristina

2019 That Time the Society for American Archaeology Blocked Me on Twitter. Powered by Osteons (blog), el 26 de abril. https://www.poweredbyosteons.org/2019/ 04/that-time-society-for-american.html, accedido el 25 de septiembre de 2020. 
Klembara, Nathan y Patricia Markert

2019 \#SAA2019 and the Public Face of Harassment: Thoughts and Resources on \#metoo and the SAA. MAPA. Master of Arts in Public Archaeology at Binghamton University (website), el 1 de mayo. http:/ mapabing.org/2019/05/01/saa2019-and-the-public-faceof-harassment-thoughts-and-resources-on-metoo-andthe-saa/?fbclid=IwAR2uOueyvC7z9nngfPRmWlwwC2 NaTRu7eOjG6wAS23Mk1zJ4hfeo7XFhNMo, accedido el 25 de septiembre de 2020.

Kloß, Sinah Theres

2017 Sexual(ized) Harassment and Ethnographic Fieldwork: A Silenced Aspect of Social Research. Ethnography 18:396-414.

Lavery, Michelle

2014 On Sexual Harassment and Fieldwork: Being an Ally. GradHacker: Inside Higher Ed (blog), el 21 de agosto. https://www.insidehighered.com/blogs/gradha cker/sexual-harassment-and-fieldwork-being-ally, accedido el 9 de enero de 2020.

Leighton, Mary

2020 Myths of Meritocracy, Friendship, and Fun Work: Class and Gender in North American Academic Communities. American Anthropologist 122:444-458.

Levine, Mary Ann

1994 Presenting the Past: A Review of Research on Women in Archaeology. En Equity Issues for Women in Archaeology, editado por Sarah M. Nelson, Margaret C. Nelson y Alison Wylie, pp. 23-36. Archaeological Papers 5. American Anthropological Association, Washington, DC.

Marín-Spiotta, Erika, Blair Schneider y Mary Anne Holmes

2016 Steps to Building a No-Tolerance Culture for Sexual Harassment. EOS: Earth and Space Science News, el 28 de enero. https://eos.org/opinions/steps-to-building-ano-tolerance-culture-for-sexual-harassment, accedido el 16 de enero de 2020.

Meyers, Maureen S., Tony Boudreaux, Stephen Carmody, Victoria Dekle, Elizabeth Horton y Alice Wright

2015 Preliminary Results of the SEAC Sexual Harassment Survey. Horizon and Tradition 57(1):19-35.

Meyers, Maureen S., Elizabeth T. Horton, Edmond A. Boudreaux, Stephen B. Carmody, Alice P. Wright y Victoria G. Dekle

2018 The Context and Consequences of Sexual Harassment in Southeastern Archaeology. Advances in Archaeological Practice 6:275-287.

Moser, Stephanie

2007 On Disciplinary Culture: Archaeology as Fieldwork and Its Gendered Associations. Journal of Archaeological Method and Theory 14:235-263.

National Academies of Sciences, Engineering, and Medicine (NASEM)

2018 Report Release Presentation-Sexual Harassment of Women: Climate, Culture, and Consequences in Academic Sciences, Engineering, and Medicine. Documento electrónico, https://sites.nationalacademies.org/cs/groups/ shstudysite/documents/webpage/shstudy_187268.pdf, accedido el 27 de enero de 2020.

National Sexual Assault Coalition Resource Sharing Project and National Sexual Violence Resource Center (NSACRSP/NSVRC)

2017 Building Cultures of Care: A Guide for Sexual Assault Services Programs. Documento electrónico, https://www.nsvrc.org/publications/nsvrc-publicationsguides/building-cultures-care-guide-sexual-assault-ser vices-programs, accedido el 21 de septiembre de 2020.
Nelson, Robin G., Julienne N. Rutherford, Katie Hinde y Kathryn B. H. Clancy

2017 Signaling Safety: Characterizing Fieldwork Experiences and Their Implications for Career Trajectories. American Anthropologist 119:710-722.

Nelson, Sarah Milledge, Margaret C. Nelson y Alison Wylie (editores)

1994 Equity Issues for Women in Archaeology. Archaeological Papers 5. American Anthropological Association, Washington, DC.

Quinlan, Liz

2019 Timeline of Events-SAA 2019: Summary of David Yesner Situation and SAA Executive Board Actions. Documento electrónico, https://docs.google. com/document/d/1gxa-BYj-dTeCb7w5LLCTUbEwm 2v4NyE2uIuCCLy181w/edit, accedido el 25 de septiembre de 2020.

Radde, Hugh D.

2018 Sexual Harassment among California Archaeologists: Results of the Gender Equity and Sexual Harassment Survey. California Archaeology 10:231-255.

Richard, Lucie, Lise Gauvin y Kim Raine

2011 Ecological Models Revisited: Their Uses and Evolution in Health Promotion over Two Decades. Annual Review of Public Health 32:307-326.

Rivera, Daniella

2019 Open Letter Gathers 1,800-Plus Signatures Asking Organization to Bar Ex-UAA Professor. KTVA.com, el 16 de abril. https://www.ktva.com/story/40311625/ open-letter-gathers-1800plus-signatures-asking-organi zation-to-bar-exuaa-professor, accedido el 4 de diciembre de 2019.

Rocks-Macqueen, Doug

201891 Stories of Archaeology. Doug's Archaeology: Investigating the Profession and Research (blog), el 14 de abril. https://dougsarchaeology.wordpress.com/ 2018/04/14/91-stories-of-archaeology/, accedido el 5 de febrero de 2020 .

She

2000 Sex and a Career. World Archaeology 32:166-172. Society for American Archaeology (SAA)

2015 SAA Background and Resource Guide for Addressing Harassment and Violence. Documento electrónico, https://documents.saa.org/container/docs/default-source/ doc-careerpractice/harassment_resource.pdf?sfvrsn $=\mathrm{d} 5 \mathrm{~b}$ 7b7d8_4, accedido el 9 de enero de 2020.

2020a Society for American Archaeology Meeting Safety Policy and Code of Conduct at SAA Events. Documento electrónico, https://www.saa.org/annualmeeting/submissions/anti-harassment-policy, accedido el 6 de enero de 2020.

$2020 \mathrm{~b}$ Welcome to the SAA. Documento electrónico, https://www.saa.org/quick-nav/about-saa, accedido el 28 de septiembre de 2020.

Souleles, Daniel

2020 What to Do with the Predator in Your Bibliography. Allegralab (blog), el 15 de septiembre. https://allegralabo ratory.net/what-to-do-with-the-predator-in-your-bibliography/, accedido el 16 de septiembre de 2020.

Southeastern Archaeological Conference (SEAC)

2018 SEAC Policy Statement on Sexual Harassment and Sexual Assault. Documento electrónico, https://www. southeasternarchaeology.org/sexual-harassment-task-force/, accedido el 9 de enero de 2020.

2019 SEAC Task Force on Sexual Harassment and Assault. Documento electrónico, https://www.southeasternarchae 
ology.org/sexual-harassment-task-force/, accedido el 23 de noviembre de 2020.

Speier, Jackie

2016 Congresswoman Jackie Speier Introduces Bill to Stop Rampant Sexual Abuse, Harassment in STEM Research (news release), el 21 de septiembre. Documento electrónico, https://speier.house.gov/2016/9/ congresswoman-jackie-speier-introduces-bill-stop-rampantsexual-abuse, accedido el 28 de septiembre de 2020.

Substance Abuse and Mental Health Services Organization (SAMHSA)

2014 SAMHSA's Concept of Trauma and Guidance for a Trauma-Informed Approach. HHS Publication No. (SMA) 14-4884. Substance Abuse and Mental Health Services Administration, Rockville, Maryland.

Tello, Monique

2018 Trauma-Informed Care: What It Is, and Why It's Important. Harvard Health Blog (blog), el 26 de octubre. https://www.health.harvard.edu/blog/trauma-informedcare-what-it-is-and-why-its-important-2018101613562, accedido el 8 de septiembre de 2020.

University at Buffalo, Buffalo Center for Social Research

2021 What Is Trauma-Informed Care? Documento electrónico, http://socialwork.buffalo.edu/social-research/ institutes-centers/institute-on-trauma-and-trauma-informedcare/what-is-trauma-informed-care.html, accedido el 9 de febrero de 2021.

van der Vaart-Verschoof, Sasja

2019 \#Metoo: A Woman in Academia and Archeology. Overdressed Archeologist (blog), el 6 de abril. http:// vandervaart-verschoof.com/metoo-a-woman-in-academiaarcheology/, accedido el 30 de enero de 2020.

VanDerwarker, Amber M., Kaitlin Brown, Toni Gonzalez y Hugh D. Radde

2018 The UCSB Gender Equity Project: Taking Stock of Mentorship, Equity, and Harassment in California Archaeology through Qualitative Survey Data. California Archaeology 10:131-158.

Voss, Barbara L.

2021 Documentación de culturas de acoso en la arqueología: Revisión y análisis de estudios de investigación cuantitativa y cualitativa. Latin American Antiquity 32, in press. DOI:10.1017/laq.2021.79.

Wade, Lizzie

2019 \#MeToo Controversy Erupts at Archaeology Meeting. ScienceMag.org (blog), el 15 de abril. https:/l www.sciencemag.org/news/2019/04/metoo-controversyerupts-archaeology-meeting, accedido el 3 de diciembre de 2019.

Walter, Holly y Kersten Bergstrom

2020 A Long Journey Home: Faculty Guide. Documento electrónico, https://metooanthro.org, accedido el 2 de septiembre de 2020.

Wood, Bernard

2015 Zero Tolerance. Period. Science 350:487.

Wright, Rita P.

2003 Gender Matters: A Question of Ethics. En Ethical Issues in Archaeology, editado por Larry J. Zimmerman, Karen D. Vitelli y Julie Hollowell-Zimmer, pp. 225237. AltaMira, Walnut Creek, California.

Wylie, Alison

1994 The Trouble with Numbers: Workplace Climate Issues in Archaeology. Originalmente publicado en Women in Archaeology: A Feminist Critique, editado por Hilary du Cros y Laurajane Smith, Australian National University, Canberra 1993. En Equity Issues for Women in Archaeology, editado por Sarah M. Nelson, Margaret C. Nelson y Alison Wylie, pp. 65-71. Archaeological Papers No. 5. American Anthropological Association, Washington, DC.

Wylie, Alison y Margaret C. Nelson

1994 Preface. En Equity Issues for Women in Archaeology, editado por Sarah M. Nelson, Margaret C. Nelson y Alison Wylie, pp. ix-xii. Archaeological Papers 5. American Anthropological Association, Washington, DC.

Submitted July 2, 2021; Accepted August 19, 2021 